\title{
Prevalence of Minimal Hepatic Encephalopathy among Chronic Hepatitis C Patients and Assessment of their Response to Lactulose
}

\author{
Mahmoud M. Sheded', Fawzy A. KaliI', Yossri A.Ashour ${ }^{3}$ \\ Departments of ${ }^{1}$ Infectious \& Endemic disease, ${ }^{2}$ Internal Medicine, and ${ }^{3}$ Neurology, Faculty of Medicine, Suez \\ Canal University, Egypt.
}

\begin{abstract}
Background: Minimal hepatic encephalopathy (MHE) impairs quality of life and predicts overt hepatic encephalopathy in cirrhotic patients. Diagnosis of MHE requires cumbersome tests. Lactulose is effective in the treatment of MHE. Aim: This study aimed to detect the prevalence of (MHE) among chronic hepatitis $\mathrm{C}$ patients and assessment of the response of MHE patient's to treatment with lactulose after one month. Patients and Methods: One hundred and fifty six patients were evaluated by psychometry (number connection tests A, B or figure connection tests $A, B$ ), venous ammonia, and critical flicker frequency (CFF). MHE was diagnosed by abnormal psychometry (>2SD age matched controls) and abnormal CFF (CFF was considered abnormal when the value was $<39 \mathrm{~Hz}$ ). MHE patients were treated with lactulose for one month. Response was defined by normalization (<2SD of matched controls) of both psychometry and CFF ( $>39 \mathrm{~Hz}$ ). Results: Of the 156 patients (age was 44.6 \pm 12.3 years, $M: F$ was 52.8:47.2), Child Turcott Pugh groups A:B:C were 47:31:22, 116 (74.4\%) had abnormal results of psychometric tests, and 111 (71.2\%) had abnormal CFF. One hundred and two (65.4\%) patients were diagnosed as having MHE. Child groups A:B:C among thus patients was 33:37:30. The percentage of MHE in different Child groups A, B \& C was 46.6\%, $79.2 \% \& 86 \%$ respectively. After treatment for one month, 69 (67.6\%) patients recovered, while 33 (32.3\%) continued to have MHE. Conclusion: MHE is prevalent among patient with CHC especially among child $B$ and $C$ groups of patients. Lactulose is effective in the treatment of MHE.
\end{abstract}

Keywords: HCV, critical flicker frequency, psychometric tests

\section{Introduction}

MHE has been described previously using several different names, such as, early, lowgrade, latent or subclinical, HE to identify patients with subtle cognitive function $a b-$ normalities $^{(1,2) \cdot}$ In 1970, Zeegen et al ${ }^{(3)}$ were the first to describe this condition, when they discovered that $38 \%$ of patients who had undergone portal decompression surgery scored abnormal in the Reitan trailmaking test (number connection test). Eight years later, the term subclinical HE was introduced to describe patients with abnormal psychometric tests and an abnormal $\mathrm{EEG}^{(4)}$. In recent years, the term MHE has been preferred to latent, preclinical or subclinical HE, which may mislead by indicating that the condition is below the threshold of significance ${ }^{(5,6)}$. Minimal hepatic encephalopathy (MHE) is the mildest form of spectrum of hepatic encephalopathy (HE). Patients with MHE have no recognizable clinical symptoms of HE but have mild cognitive and psychomotor deficits. There are no accurate data on the inci- 
dence of $\mathrm{HE}^{(5,6)}$. However, several studies suggest that the majority of patients with cirrhosis will develop some degree of $\mathrm{HE}$ at some point during the course of disease. Overt HE occurs in approximately 30\% to $50 \%$ of cirrhotic patients ${ }^{(7)}$ and $10-50 \%$ of patients with transjugular intrahepatic portosystemic shunt (TIPS) $)^{(7-9)}$. The prevalence of MHE is high in patients with cirrhosis of liver and varies between $30 \%$ and $84 \%$; it is higher in patients with poor liver function. The diagnosis of HE has traditionally been linked to patients with cirrhosis of liver. However, impairment of cognitive function has been shown in patients with non cirrhotic portal fibrosis ${ }^{(10)}$ and extra hepatic portal venous obstruction and has been related to portosystemic shunting ${ }^{(11)}$.

The diagnostic criteria for MHE have not been standardized but rest on careful patient history and physical examination, normal mental status examination, demonstration of abnormalities in cognition and/or neurophysiological function, and exclusion of concomitant neurological disorders. Various tools have been evaluated for the diagnosis of MHE and include the neuropsychological tests, computerized tests $^{(12)}$, short neuropsychological, computerized test batteries, and neurophysiological tests. Regional cerebral blood flow changes, magnetic resonance imaging, and spectroscopy ${ }^{(13,14)}$, though useful for understanding pathogenic mechanisms, are currently not considered of diagnostic value. There is no ideal test for the diagnosis of MHE. However, the diagnosis of MHE requires a normal mental status examination and impairment in the performance of at least two of the following tests: number connection test-A (NCT-A), number connection Test-B (NCT-B), block design test (BDT) and digit symbol test (DST) ${ }^{(5)}$, also the use of [PSE-Syndrome-Test or psychometric hepatic encephalopathy score (PHES)], a standardized test battery includ- ing (NCT-A) and $B$, the line-tracing test (LTT), the serial-dotting test (SDT), and $\mathrm{DST}^{(15,16)}$. When possible, quantitative neurophysiologic tools (like EEG with mean dominant frequency, P300 auditory evoked potentials $)^{(12)}$ should be used. Critical flicker frequency (CFF) and inhibitory control test (ICT) are recent additions to the tests for the diagnosis of MHE. CFF tests the ability of a patient to perceive flickering and its fusion threshold. Two recent studies that evaluated its utility in the diagnosis of MHE found it to be a simple, reliable and accurate method for the diagnosis of MHE, and to be independent of age, education or training $^{(17-19)}$.

MHE is associated with impaired healthrelated quality of life, predicts the development of overt HE and is associated with poor survival. Hence, screening all patients with cirrhosis for MHE using psychometric tests, and treatment of those patients diagnosed to have MHE have been recommended $^{(15,16,)}$. In a landmark study, Wein and colleagues ${ }^{(20)}$ found that fitness to drive a car was impaired in cirrhotic patients with MHE. Patients without MHE scored similar to controls. Increased risk of automobile accidents is related to a decline in cognitive function. Bajaj et $a^{(21)}$ reported a higher self-reported occurrence of violations and accidents in patients with cirrhosis and MHE compared to healthy volunteer. Navigation is a complex activity required for safe driving and is dependent on functioning working memory, attention, and speed of mental processing. Impaired navigation skills correlate with impairment in response inhibition and attention. $\mathrm{Pa}$ tients with cirrhosis and MHE also pose navigation difficulty ${ }^{(22)}$.

Ammonia plays a key role in the pathogenesis of $\mathrm{MHE}^{(23-27)}$. Various treatment modalities have been tried for MHE, including dietary protein manipulation ${ }^{(29)}$, branched-chain amino acids ${ }^{(30,31)}$, lactu- 
lose ${ }^{(32-37)}$, flumazenil ${ }^{(38)}$, L-ornithine Laspartate $^{(39)}$, acetyl Lcarnitine ${ }^{(40)}$, and probiotics/synbiotics ${ }^{(41-43)}$. A majority of these attempts were aimed at reducing blood ammonia level, and most studies have shown improvement in psychometric measurements, ammonia levels, cerebral edema and HRQoL.

\section{Patients and Methods}

This study was conducted in the Communicable Disease Research and Training Center (CDRTC) in Suez city in the period from March 2011 until October 2011. Informed consent was obtained and the research protocol was approved by the ethics committee of the hospital in accordance with the ethical guidelines of the 1975 Declaration of Helsinki.

\section{Patients}

One hundred and fifty six consecutive chronic hepatitis $\mathrm{C}$ ( $\mathrm{CHC}$ ) patients attending Communicable Disease Research and Training Center (CDRTC) in Suez city from March 2011 to October 2011, without HE were screened for MHE. CHC was diagnosed on a clinical basis involving HCV Ab, laboratory tests, endoscopic evidence, and sonographic findings. The exclusion criteria were: 1 ) the presence of overt HE or a history of HE at enrollment, 2) a history of taking lactulose or any antibiotics, 3) alcohol intake, gastrointestinal hemorrhage or spontaneous bacterial peritonitis during the past 12 weeks, 4) previous TIPS or shunt surgery, 5) significant co-morbid illness such as heart, 6) respiratory or renal failure, and 7) any neurologic diseases (such as Alzheimer's disease, Parkinson's disease and non hepatic metabolic encephalopathies, patients on psychoactive drugs such as antidepressants or sedatives and antiepileptic drugs, also Patients with color blindness, mature cataract or diabetic retinopathy were also excluded).

\section{Study maneuver}

All patients were subjected to the following: Revision of patients' files: Full History taking and Clinical examination: the clinical, biochemical (After overnight fasting, venous blood was taken for routine liver function and hematological tests, i.e. $C B C$, ALT, AST, total and direct bilirubin, albumin, prothrombin time and creatinine, imaging (abdominal ultrasound) and Venous ammonia was assessed by the ammonia checker II) before and after treatment for one month. Psychometric testing: All patients underwent a series of psychometric tests including number connection tests (NCT-A, NCT-B) if literate, and figure connection tests (FCT-A, FCT-B) if illiterate. Four parallel forms are available for both NCT and FCT, and we used 2 of them to avoid learning effects, one at the start, and the other at the end. The test score is the time required to complete the test, including the time needed to correct any errors. The results of tests were considered abnormal when test scores were more than the mean +2SD from age and educationmatched controls ${ }^{(15,16)}$. The result of a psychometric test was considered abnormal when the results of both NCT-A and NCT-B or FCT-A and FCT-B were abnormal.

\section{Measurement of CFF threshold}

CFF threshold was used to measure visual discrimination and general arousal. CFF was determined with a HEPAtonorm analyzer at the bedside. Patients were first instructed and trained about the procedure. Flicker frequencies were measured 6 times and the mean value was calculated. CFF was considered abnormal when the value was $<39 \mathrm{~Hz}^{(17-19)}$. The CFF thresholds and psychometric measurements were determined on the same day. It was measured at the 
time of enrollment and after one month of treatment (4 weeks treatment and measurement directly after the last lactulose administration).

\section{Treatment}

Patients were given $30-40 \mathrm{ml}$ lactulose per day. Compliance with the therapy was assured primarily by ensuring increased stool frequency and a change to a softer consistency.

\section{Statistical analysis}

Data are presented as mean \pm SD. Statistical analysis was made using Student's paired $t$ test and Fisher's exact test. Correlations between different tests were calculated by Spearman's rank-order correlation coefficient. A significance level of $\leq 0.05$ was used in all analyses. Statistical analyses were made using the software SPSS version 10.0 (SPSS, Chicago, IL).

\section{Results}

Of the 156 patients included in this study (age was $44.6 \pm 12.3$ years, $M: F$ was 52.8:47.2) (Table 1); (Child Turcott Pugh groups A:B:C were 47:31:22, 102 (65.4.\%) patients were diagnosed with MHE. Baseline characteristics of MHE and non-MHE patients were compared (Table 2). Child Turcott Pugh groups A:B:C among thus patients was $33: 37: 30$. The percentage of MHE among different Child Turcott Pugh groups A, B \&C patients were $46.6 \%, 79.2 \%$ \& $86 \%$ respectively. MHE was significantly higher among patients with Child score $C$ and $\mathrm{B}$ compared to patients with Child A score. One hundred and two patients were followed-up for one month and both psychometry and CFF were determined immediately after 4 weeks of treatment. Sixty-nine $(67.6 \%)$ of them recovered, while $33(32.3 \%)$ continued to have MHE (abnor- mal results of psychometric or (FF tests) (Table 3).

Table 1: Demographic, clinical, and biochemical characteristics of the study group $(n=156)$

\begin{tabular}{|l|l|}
\hline Characteristics & Values \\
\hline Age (years) & $44.6 \pm 12.3$ \\
Sex (M/F) & $52.8 / 47.2$ \\
Educational status & \\
$\quad$ Illiterate & $41(26.3)$ \\
$\quad \leq 12$ year education & $91(58.3)$ \\
$>12$ year education & $24(15.4)$ \\
ALT (U/L) & $44.7 \pm 29.1$ \\
AST (U/L) & $51.5 \pm 33.8$ \\
S. Albumin(g/dl) & $3.6 \pm 2.1$ \\
S. Total Bilirubin (mg/dl) & $2.8 \pm 1.4$ \\
INR & $1.1 \pm 0.6$ \\
Ammonia ( $\mu$ mol/L) & $91.9 \pm 33.5$ \\
Child A, $n(\%)$ & $73(47 \%)$ \\
Child B, $n(\%)$ & $48(31 \%)$ \\
Child C, $n(\%)$ & $35(22 \%)$ \\
\hline
\end{tabular}

Data are presented as mean \pm SD; INR= international normalization ratio; $\mathrm{ALT}=$ alanine aminotransferase; AST=Aspartate aminotransferase

\section{Psychometric tests}

Out of the 156 patients, 41 were illiterate, 91 were sub-graduates ( $\leq 12$ years of education), and 24 were graduates ( $>12$ years of education). Of all patients, $115(73.7 \%)$ could have NCT and 41 (26.4\%) had FCT due to illiteracy. One hundred and sixteen patients (74.4\%) had abnormal results of psychometric tests, and 12 (7.7\%) patients with abnormal results of psychometric tests had normal CFF. After one-month treatment of MHE, the results of psychometry were abnormal in 33 (21.2\%).

CFF test

Among the studied patients, the mean CFF was $43.8 \pm 5.1$. CFF was abnormal $(>39 \mathrm{~Hz})$ in 111 (71.2\%) patients. After one-month treatment of MHE, the results of CFF was abnormal in 30 (19.2\%). CFF significantly correlated with psychometric tests, Child score, and venous ammonia, before and after treatment (Table 4). 
Table 2: Demographic, clinical, and biochemical characteristics of patients with and without MHE

\begin{tabular}{|l|lll|}
\hline Baseline parameters & $\begin{array}{l}\text { MHE } \\
(n=102)\end{array}$ & $\begin{array}{l}\text { Non-MHE } \\
(n=54)\end{array}$ & $P$ \\
\hline Age (years) & $42.6 \pm 11.3$ & $40.3 \pm 12.1$ & 0.30 \\
Sex (M/F) & $49 / 11$ & $33 / 17$ & 0.70 \\
CFF(Hz) & $34.2 \pm 2.7$ & $43.8 \pm 5.1$ & $0.001^{*}$ \\
Psychometric tests (sec) & & & \\
$\quad$ NCT-A & $59.2 \pm 10.1$ & $31.8 \pm 14$ & 0.5 \\
NCT-B & $163.3 \pm 19.7$ & $89.4 \pm 41$ & 0.1 \\
FCT-A & $77.8 \pm 13.8$ & $40.7 \pm 16.8$ & $0.001^{*}$ \\
FCT-B & $161.9 \pm 13.9$ & $74.5 \pm 47$ & 0.2 \\
Ammonia ( $\mu$ mol/L) & $98.4 \pm 29.1$ & $68.5 \pm 24.9$ & $0.001^{*}$ \\
Child score & $9.1 \pm 2.9$ & $7.0 \pm 1.9$ & $0.003^{*}$ \\
Child A, $n(\%)$ & $34(46.6 \%)$ & $39(53.4 \%)$ & $0.001^{*}$ \\
Child B, $n$ (\%) & $34(79.2 \%)$ & $14(20.8 \%)$ & \\
Child C, $n$ (\%) & $30(80 \%)$ & $5(20 \%)$ & \\
\hline
\end{tabular}

$\mathrm{MHE}=$ minimal hepatic encephalopathy; $\mathrm{CFF}=$ critical flicker frequency; $\mathrm{NCT}=$ number connection test; $\mathrm{FCT}=$ figure connection test; Data are presented as mean \pm SD.

$*=0 \leq 0.05$ (statistically significant).

Table 3: Treatment response in MHE patients

\begin{tabular}{|l|lll|}
\hline \multicolumn{1}{|c|}{ Parameters } & $\begin{array}{l}\text { MHE } \\
(n=102)\end{array}$ & $\begin{array}{l}\text { After treatment } \\
(n=102)\end{array}$ & $P$ \\
\hline CFF $(\mathrm{Hz})$ & $34.2 \pm 2.7$ & $41.5 \pm 4.4$ & $0.001^{*}$ \\
Psychometric tests $(\mathrm{sec})$ & & & \\
NCT-A & $59.2 \pm 10.1$ & $31.9 \pm 10.4$ & 0.5 \\
NCT-B & $163.3 \pm 19.7$ & $71.2 \pm 35.6$ & 0.4 \\
FCT-A & $77.8 \pm 13.8$ & $41.9 \pm 13.3$ & $0.007^{*}$ \\
FCT-B & $161.9 \pm 13.9$ & $77.8 \pm 44$ & 0.8 \\
Venous ammonia ( $\mu$ mol/L) & $98.4 \pm 29.1$ & $73.8 \pm 29.5$ & $0.001^{*}$ \\
Child score & $9.1 \pm 2.9$ & $8.1 \pm 1.5$ & 0.390 \\
\hline
\end{tabular}

$\mathrm{MHE}=$ minimal hepatic encephalopathy, $\mathrm{CFF}=$ critical flicker frequency; $\mathrm{NCT}=$ number connection test; $\mathrm{FCT}=$ figure connection test; Data are presented as mean $\pm \mathrm{SD} .{ }^{*} p$ $\leq 0.05$ is statistically significant

\section{Discussion}

In this study, the prevalence of MHE among CHC patients was $65.4 \%$. CFF alone diagnosed MHE in $71.2 \%$ of patients, and psychometric tests diagnosed MHE in $74.4 \%$ of patients. Various methods, including neurophysiological or psychometric test or in combination, have been used in the diagnosis of $\mathrm{MHE}^{(5,15,16)}$. Among these methods NCT (A, B) is commonly used for detecting MHE, but this test may overdiagnose if corrections for age and educational status are not applied ${ }^{(5,15,16)}$.
Illiterate people and those unfamiliar with roman alphanumeric notations, especially in deve-lopping countries, cannot perform NCT. FCT is based on the subject's identification of figures rather than the alphabet or numerals. FCT is as useful as NCT in detecting psychomotor performance defects in a large cohort of cirrhotic patients without overt encephalo-pathy $(44,45)$. In this study, 41 patients could not perform NCT due to illiteracy; hence, FCT is an important test in these patients. 
Table 4: Correlation ( $r$ ) of CFF to psychometry, child score, and venous ammonia (Before and after treatment)

\begin{tabular}{|l|llll|}
\hline Parameters & \multicolumn{2}{|c}{ Before treatment } & \multicolumn{2}{c|}{ After treatment } \\
& $(r)$ & $p$ & $(r)$ & $p$ \\
\hline NCT-A (sec) & -0.434 & $0.001^{*}$ & -0.325 & 0.049 \\
NCT-B (sec) & -0.425 & $0.001^{*}$ & -0.222 & 0.287 \\
FCT-A (sec) & -0.305 & 0.310 & -0.380 & 0.190 \\
FCT-B (sec) & -0.311 & 0.381 & -0.229 & 0.417 \\
Child score & -0.490 & $0.001^{*}$ & -0.493 & $0.001^{*}$ \\
Venous ammonia ( $\mu$ mol/L) & -0.452 & $0.001^{*}$ & -0.552 & $0.001^{*}$ \\
\hline \multicolumn{7}{l}{ MHE= minimal hepatic encephalopathy, CFF= critical flicker frequency; NCT= number } \\
connection test; FCT = figure connection test, * $p \leq 0.05$ is statistically significant
\end{tabular}

Using the same variant for follow-up of MHE patients may give a false impression of improvement, but this can be circumvented if a different variant is used, as we did in this study. In addition, no single psychometric test can pick up MHE reliably ${ }^{(44,45)}$.

Although the majority of previous studies agreed that MHE is a significant problem requiring testing, and there is actually a problem in the diagnosis of MHE as part of regular clinical practice because of lack of standardization of diagnostic tests and its normal values, and the time needed to do a battery of tests ${ }^{(15,16,46)}$. Evaluation of cortical and sub cortical cognitive function, complicating factors such as movement disorders may give a false impression of significant cognitive deficits in patients with reduced peripheral motor skills needed for psychometric tests $^{(15,16,44)}$.

CFF is a well-established neurophysiological technique that measures the ability of the central nervous system to detect flickering light, which is directly influenced by cortical activity ${ }^{(17)}$. CFF appears to detect a broad spectrum of neuro-psychological abnormalities ranging from visual signal processing (retinal gliopathy) to cognitive functions ${ }^{(47)}$. In recent years, various studies have shown CFF in the diagnosis of $\mathrm{MHE}^{(15,16,45)}$. In this study CFF significantly correlated with the other used tests in the diagnosis of MHE before and after treat- ment ${ }^{(15,16)}$, psychometric tests NCT-A, child score and Venous ammonia level $(p=0.001)$ in the diagnosis of MHE and assessment of its recovery. These relation-ships suggest that CFF measurements can used as a specific and sensitive test for diagnose MHE in cirrhotic patients. It can be administered easily with relatively little training to the doctors and patient, and does not show a learning effect ${ }^{(15,16)}$. Ammonia has been shown to be an important etiological parameter in the pathogenesis of $\mathrm{MHE}^{(23-27)}$. In this study, venous ammonia was significantly higher in MHE patients than in non-MHE patients $(p=0.001)$.

In this study there was also significantly higher prevalence of MHE in child B and C patients, while previous studies suggested that the neurological abnormality in cirrhotic patients has little or no relationship with the degree of liver failure, but is correlated with disturbances in nitrogen metabolism ${ }^{(23,26)}$. We also demonstrated a significant correlation of venous ammonia level with CFF, in accord with Kircheis et $\mathrm{al}^{(21)}$. Lactulose is an effective treatment for MHE. In this study, lacunose also improved MHE in $67.6 \%$ of patients, and all patients tolerated it well without any noticed side effects. Few studies used psychometric tests in assessment of response to treatment with lactulose ${ }^{(35)}$, because there are no data validating their use in a serial longitudinal 
manner, also age, education, and learning effects may adversely affect the results. Similarly, some studies used methods like brain stem auditory evoked potential and P300ERP to assess recovery from MHE $(33,34,37)$. Though these tests are more objective and do not show learning effects, they are expensive, difficult to perform and cannot be done at the bedside without trained personnel and standardization ${ }^{(36)}$. A limitation of this study is the lack of a placebo limb for the management of MHE and evaluating diagnostic tests for the same group.

\section{Conclusions}

MHE is highly prevalent among patients with $\mathrm{CHC}$ especially with child $\mathrm{B}$ and $\mathrm{C}$ and assessment of patients in these child groups is highly recommended. MHE is associated with significant disability and poor HRQOL. An early identification of MHE may improve the HRQOL and the prognosis of these patients. CFF is a simple, relatively reliable test for the diagnosis of MHE and assessment of its recovery. We highly recommend the use of this test for assessment of drivers before approving them for having a driver license. Lactulose is highly effective, economic and a well tolerated treatment for MHE.

\section{References}

1. Rikkers L, Jenko P, Rudman D, Freides D. Subclinical hepatic encephalopathy: detection, prevalence, and relationship to nitrogen metabolism. Gastroenterology 1978; 75 (3):462-469.

2. Schomerus $\mathrm{H}$, Hamster $\mathrm{W}$, Blunck $\mathrm{H}$, Reinhard U, Mayer K, Dölle W. Latent portosystemic encephalopathy. I. Nature of cerebral function defects and their effect on fitness to drive. Dig Dis Sci 1981; 26(7):622630.

3. Zeegen R, Drinkwater JE, Dawson AM. Method for measuring cerebral dysfunction in patients with liver disease. $\mathrm{Br}$ Med $\mathrm{J}$ 1970;2(5710):633-636.

4. Ferenci P, Lockwood A, Mullen K, Tarter R, Weissenborn K, Blei AT. Hepatic encephalopathy definition, nomenclature, diagnosis, and quantification: final report of the working party at the 11th World Congresses of Gastroenterology, Vienna, 1998. Hepatology 2002; 35 (3):716-721.

5. Mullen KD. Review of the final report of the 1998 Working Party on definition, nomenclature and diagnosis of hepatic encephalopathy. Aliment Pharmacol Ther 2007;25 Suppl 1:11-16.

6. Amodio P, Del Piccolo F, Petteno E, et al. Prevalence and prognostic value of quantified electroencephalogram (EEG) alterations in cirrhotic patients. J Hepatol 2001;35 (1):37-45

7. Nolte W, Wiltfang J, Schindler C, et al. Portosystemic hepatic encephalopathy after transjugular intrahepatic portosystemic shunt in patients with cirrhosis: clinical, laboratory, psychometric, and electroencephalographic investigations. Hepatology 1998; 28 (5):1215-1225

8. Boyer TD, Haskal ZJ, AASLD. The role of transjugular intrahepatic portosystemic shunt in the management of portal hypertension. Hepatology 2005;41(2):386400.

9. Sarin SK, Nundy S. Subclinical encephalopathy after portosystemic shunts in patients with non-cirrhotic portal fibrosis. Liver 1985;5(3):142-146.

10. Mínguez B, García-Pagán JC, Bosch J, Turnes J, Alonso J, Rovira A, Córdoba J. Noncirrhotic portal vein thrombosis exhibits neuropsychological and MR changes consistent with minimal hepatic encephalopathy. Hepatology 2006; 43(4):707-714.

11. Sharma P, Sharma BC, Puri V, Sarin SK. Minimal hepatic encephalopathy in patients with extrahepatic portal vein obstruction. Am J Gastroenterol 2008;103 (6):1406-1412.

12. Weissenborn K, Ennen JC, Schomerus H, Rückert N, Hecker H. Neuropsychological characterization of hepatic encephalopathy. J Hepatol 2001;34 (5):768-773.

13. Venktaramarao SH, Mittal, Prabhakar S, Dhiman RK. Brain perfusion single photon 
emission computed tomography (SPECT) abnormalities in patients with minimal hepatic encephalopathy (abstract). J Gastroenterol Hepatol 2008; 23 (Suppl 5): A62.

14. Grover VP, Dresner MA, Forton DM, et al. Current and future applications of magnetic resonance imaging and spectroscopy of the brain in hepatic encephalopathy. World $J$ Gastroenterol 2006;12 (19):2969-2978.

15. Dhiman RK, Saraswat VA, Verma M, Naik SR. Figure connection test: a universal test for assessment of mental state. J Gastroenterol Hepatol 1995;10 (1):14-23.

16. Bajaj JS, Etemadian A, Hafeezullah M, Saeian $K$. Testing for minimal hepatic encephalopathy in the United States: An AASLD survey. Hepatology 2007;45(3):833-834.

17. Romero-Gómez M, Córdoba J, Jover R, et al. Value of the critical $\mathrm{fl}$ icker frequency in patients with minimal hepatic encephalopathy. Hepatology 2007;45 (4):879-885.

18. Kircheis G, Wettstein M, Timmermann L, Schnitzler A, Häussinger D. Critical flicker frequency for quantification of low grade hepatic encephalopathy. Hepatology 2002;35 (2):357-366.

19. Sharma P, Sharma BC, Puri V, Sarin SK. Critical flicker frequency: diagnostic tool for minimal hepatic encephalopathy. J Hepatol 2007;47(1):67-73.

20.Wein C, Koch H, Popp B, Oehler G, Schauder $P$. Minimal hepatic encephalopathy impairs fitness to drive. Hepatology 2004;39(3):739745 .

21. Kircheis G, Knoche A, Hilger N, et al. Hepatic encephalopathy and fitness to drive. Gastroenterology 2009;137 (5):1706-1715.

22. Bajaj JS, Saeian K, Schubert CM, et al. Minimal hepatic encephalopathy is associated with motor vehicle crashes: the reality beyond the driving test. Hepatology 2009;50 (4):1175-1183.

23. Butterworth RF. Pathophysiology of hepatic encephalopathy: a new look at ammonia. Metab Brain Dis 2002;17 (4):221-227

24. Takano T, Tian GF, Peng W, et al. Astrocytemediated control of cerebral blood flow. Nat Neurosci 2006;9 (2):260-270.

25. Ahboucha S, Butterworth RF. The neurosteroid system: implication in the pathophysiology of hepatic encephalo- pathy. Neurochem Int 2008;52 (4-5):575587.

26. Balata S, Olde Damink SW, Ferguson K, et al. Induced hyperammonemia alters neuropsychology, brain MR spectroscopy and magnetization transfer in cirrhosis. Hepatology 2003;37 (4):931-939.

27. Lockwood AH, Yap EW, Wong WH. Cerebral ammonia metabolism in patients with severe liver disease and minimal HE. J Cereb Blood Flow Metab 1991;11:337-341.

28.Dhiman RK, Solanki KK. Management of hepatic encephalopathy: Seen and Unseen. In: Medicine Update Vol 17;Ed YK Munjal. 2007;pp 259-271.

29. De Bruijn KM, Blendis LM, Zilm DH, Carlen $\mathrm{PL}$, Anderson GH. Effect of dietary protein manipulation in subclinical portal-systemic encephalopathy. Gut 1983;24 (1):53-60.

30.Egberts EH, Schomerus H, Hamster W, Jürgens $P$. Branched chain amino acids in the treatment of latent portosystemic encephalopathy. A double blind placebocontrolled crossover study. Gastroenterology 1985;88 (4):887-895.

31. Plauth $M$, Egberts EH, Hamster $W$, et al. Longterm treatment of latent portosystemic encephalopathy with branched chain amino acids: a double blind placebocontrolled cross over study. J Hepatol 1993:17 (3);308-314.

32. Prasad S, Dhiman RK, Duseja A, Chawla Y, Sharma A, Agarwal R. Lactulose improves cognitive functions and health related quality of life in cirrhotic patients with minimal hepatic encephalopathy. Hepatology 2007;45(3): 549-559.

33. Morgan MY, Alonso M, Stanger LC. Lactilol and Lactulose for treatment of subclinical hepatic encephalopathy in cirrhotic patients. A randomised, cross-over study. J Hepatol 1989;8 (2):208-217.

34. Watanable A, Sakai T, Sato S, et al. Clinical efficacy of Lactulose in cirrhotic patients with and without subclinical hepatic encephalopathy. Hepatology 1997;26 (6):1410-1404. 
35. Horsmans Y. Solbreux PM, Daenens C. Desager JP, Geubel AP. Lactulose improves psychometric testing in cirrhotic patients with subclinical encephalpathy. Aliment Pharmacol Ther 1997:11(1):165-170.

36. Quero JC, Groenweg M, Muelster J, Hop WCJ, Schalm SW. Does a low dose of lactulose improve quality of life in patients with liver cirrhosis. In: Record C, Al Mardini $\mathrm{H}$, eds. Advances in Hepatic Encephalopathy \& Metabolism in Liver Disease. New Castle Upon Tyne: Medical faculty, University of New-castle upon Tyne; 1997; p. 459-465.

37. Nie YQ, Zeng Z, Li YY, Sha WH, Ping L, Dai SJ. Long-term efficacy of lactulose in patients with subclinical hepatic encephalopathy. Zhonghua Nei Ke Za Zhi.2003;42(4):261-266.

38. Amodio P, Marechtti P, Del Piccolo F, et al. The effect of flumazenil on subclinical psychometric or neurophysi-ological alterations in cirrhotic patients: a double blind placebo controlled study. Clin Physiol 1997;17(5):533-539.

39. Kircheis G, Nilius R, Held C, et al. Therapeutic efficacy of L-ornithine-Laspartate infusions in patients with cirrhosis and hepatic encephalopathy: results of a placebo-controlled, double blind study. Hepatology 1997;25 (6):13511360.

40. Malaguarnera M, Gargante MP, Cristaldi $\mathrm{E}$, et al. Acetyl-L-carnitine treatment in minimal hepatic encephalopathy. Dig Dis Sci 2008; 53(11):3018-3025.

41. Liu Q, Duon ZP, Ha DK, Bengmark S, Kurtovic J, Riordan SM. Symbiotic modula-tion of gut flora: effect on minimal hepatic encephalopathy in patients with cirrhosis. Hepatology 2004;39(5):1441-1449.

42. Bajaj JS, Saeian K, Christensen KM, Hafeezullah $M$, Varma RR, Franco J, Pleuss JA, Krakower G, Hoffmann RG, Binion DG.Probiotic yogurt for the treatment of minimal hepatic encephalopathy. Am J Gastroenterol 2008;103 (7):1707-1715.
43. Sharma P, Sharma BC, Puri V, Sarin SK. An open label randomized controlled trial of lactulose and probiotics in the treatment of minimal hepatic encephalopathy. Eur J Gastroenterol Hepatol 2008;20(6):506-511.

44. Das A, Dhiman RK, Saraswat VA, Verma $M$, Naik SR. Prevalence and natural history of subclinical hepatic encephalopathy in cirrhosis. J Gastro-enterol Hepatol 2001; 16(5): 531-535.

45. Saxena N, Bhatia M, Joshi YK, Garg PK, Tandon RK. Auditory P300 event related potentials and number connection test for evaluation of subclinical hepatic encephalopathy in patients with cirrhosis of the liver: a follow-up study. J Gastroenterol Hepatol 2001;16(3):322327.

46. Vergara-Gómez $M$, Flavià-Olivella $M$, GilPrades M, Dalmau-Obrador B, CórdobaCardona J. Diagnosis and treatment of hepatic encephalopathy in Spain: results of a survey of hepatologists. Gastroenterol Hepatol 2006;29(1):1-6.

47. Zafiris O, Kircheis G, Rood HA, Boers F, Häussinger D, Zilles K. Neural mechanism underlying impaired visual judgment in the dysmetabolic brain: an fMRI study. Neuroimage 2004; 22(2): 541-552. 
